\title{
The use of verbal protocols as data: An analysis of insight in the candle problem
}

\author{
JESSICA I. FLECK and ROBERT W. WEISBERG \\ Temple University, Philadelphia, Pennsylvania
}

\begin{abstract}
In the present study, we examined the use of verbal protocols as data in the study of the cognitive processes underlying insight. Fifty-eight Temple University undergraduates attempted to solve Duncker's (1945) candle problem either silently or while thinking aloud. Solution rates, solving times, and solution types were comparable between conditions, suggesting that verbal overshadowing (Schooler, Ohlsson, \& Brooks, 1993) did not occur when the participants attempted to solve the candle problem. Subsequent analysis of verbal protocols provided a catalogue of solutions generated by the participants, as well as empirical support for the occurrence of impasse and restructuring. Although restructuring was present in the majority of protocols, including those of the participants who later produced the box solution, the presence of impasse occurred with less frequency and was not associated with production of the box solution. These results provide information concerning how the candle problem is solved and suggest that verbalization can be used to examine how individuals solve insight problems and to evaluate existing theories of insight.
\end{abstract}

The concept of insight was initially proposed by the Gestalt psychologists (e.g., Köhler, 1969; Wertheimer, 1945/1961) to refer to a specific form of problem solving insight problem solving - that was characterized by two criteria. First, prior experience was not considered to be the critical factor in solving the problem. Indeed, the Gestalt psychologists emphasized that the transfer of past solutions to new problems could have negative effects on problem solving, as exemplified in Luchins's (1942) wellknown work on problem-solving sets and Duncker's (1945) research on functional fixedness. Second, restructuring of the problem was considered to be integral to its solution (Ohlsson, 1984). Restructuring meant that the thinker had come to perceive the problem in a way that was not obvious upon initial consideration (Scheerer, 1963).

Although the Gestalt psychologists' view of prior experience as unnecessary in insightful problem solving has been challenged (e.g., Lung \& Dominowski, 1985; Weisberg \& Alba, 1981), the concept of restructuring remains a key focus in the study of insight (e.g., Knoblich, Ohlsson, Haider, \& Rhenius, 1999; Ohlsson, 1992). Ohlsson (1992) has emphasized the insight sequence, in which restructuring is preceded by a period of impasse, wherein the solver is mentally "stuck" and unable to continue working. In response to an impasse, the problem solver may discontinue work on the problem, both behaviorally and mentally. According to Ohlsson (1992), restructuring can be a mech-

We thank Nora Newcombe, Christopher Ramey, and Alan Sockloff for helpful comments on earlier versions of this article. We also thank Naomi Starkey and Mai Vue for their assistance in coding the verbal protocols. Correspondence should be addressed to J. I. Fleck, Department of Psychology, Temple University, 1701 N. 13th Street, 6th Floor, Weiss Hall, Philadelphia, PA 19122 (e-mail: jfleck@temple.edu). anism through which problem solvers break out of impasse (see also Kaplan \& Simon, 1990).

Insights occur after the problem-solver has encountered an impasse, i.e., a mental state in which problem-solving has come to a halt; all possibilities seem to have been exhausted and the problem-solver cannot think of any way to proceed. Subjectively, his or her mind is 'blank.' Behaviorally, impasses are characterized by the cessation of problem-solving activity. . . Insight, I suggest, is the act of breaking out of an impasse. ... Without the impasse, there is no insight, only smooth progress. (Ohlsson, 1992, p. 4)

Ohlsson (1992) proposed three different forms of restructuring that could be used by a solver to break out of impasse: elaboration, reencoding, and constraint relaxation. The specific criteria for each form of restructuring are described in Table 1. It is important to note that Ohlsson's (1992) view assumes that the person who is at an impasse examines aspects of the problem that might be changed, without having any specific idea of what those changes might accomplish. Changes in the problem are considered, and then, if the person is fortunate, new methods may be seen. These processes, if successful, bring about what can be called bottom-up restructuring, because the restructuring is driven by the objects in the environment (from the "bottom up").

A number of researchers have suggested that there are fundamental differences between the processes involved in solving insight problems and those involved in solving other problems, such as analytic problems (e.g., Duncker, 1945; Lavric, Forstmeier, \& Rippon, 2000; Metcalfe \& Wiebe, 1987). It has been proposed that solutions to insight problems occur suddenly and are often independent of prior knowledge (Köhler, 1925/1959), whereas solutions to analytic problems entail a smooth progression of 
Table 1

Restructuring Coding Criteria

\begin{tabular}{lll}
\hline \multicolumn{1}{c}{ Type } & \multicolumn{1}{c}{ Ohlsson (1992) } & Present Research \\
\hline Elaboration & $\begin{array}{l}\text { Information can be added by studying the problem and } \\
\text { observing previously unnoticed features (p. 13). }\end{array}$ & $\begin{array}{l}\text { Observing a new feature/function } \\
\text { of an object. } \\
\text { Reencoding }\end{array}$ \\
$\begin{array}{l}\text { The thinker must abandon or reject some component } \\
\text { of his or her current representation (p. 13). }\end{array}$ & $\begin{array}{l}\text { Rejecting the use of one object for } \\
\text { the use of a different object. }\end{array}$ \\
Constraint relaxation & $\begin{array}{l}\text { A change in the mental representation of the goal rather } \\
\text { than of the given situation (p. 14). }\end{array}$ & $\begin{array}{l}\text { A change in the participant's } \\
\text { representation of the goal state. }\end{array}$ \\
\hline
\end{tabular}

steps (Metcalfe \& Wiebe, 1987; see the quotation above from Ohlsson, 1992) and are often solved through the application of prior knowledge. Newell and Simon (1972, p. 667) have characterized problem solving via analysis as a three-stage process, involving the compilation of alternatives, the assessment of these alternatives, and finally, the selection of the alternative that results in the greatest possible gain. Although an emotional response, referred to as the Aha! experience, often accompanies insight (Perkins, 1981), it does not accompany the solutions of analytic problems. Insight is not limited to traditional problem-solving situations but can occur when a scientist creates a new theory, an inventor constructs a new invention, or a writer drafts a new piece (Weisberg, 1993; for a review of the research on insight, see Sternberg \& Davidson, 1995).

This sharp distinction between problem solving via analysis and problem solving via insight suggests a dichotomy. That is, if the insight sequence proposed by Ohlsson (1992; i.e., impasse followed by restructuring) does not occur during problem solving but the solver nonetheless achieves the solution, that solution, ipso facto, did not occur via insight. However, other researchers (e.g., Perkins, 1981; Weisberg, 1995) have suggested that the cognitive processes involved when individuals solve problems should be partitioned into finer subgroups. For example, Perkins has found that individuals can solve insight problems via analysis and still experience the emotional reaction (i.e., the Aha! experience) that is typically associated with the experience of problem solving via insight (e.g., Metcalfe \& Wiebe, 1987). In addition, Weisberg (1995; Weisberg \& Suls, 1973) has found that participants solve insight problems through restructuring that arises from the acquisition of new information during the solving process, but without the experience of impasse. This new information leads to the modification of the solver's representation of the problem.

To fully examine the cognitive processes involved in insightful thinking, it may be necessary to expand the category of cognitive processes that fall under the classification of insightful thinking. Perkins (1981) and Weisberg (1995) have indicated that problem solving occurs via processing that is neither insightful under Ohlsson's (1992) strict definition of the insight sequence nor analytic under the strict interpretation of problem solving via analysis. In other words, the occurrence of restructuring without impasse, or the occurrence of the Aha! experience in conjunction with analysis, may warrant further examination as forms of insightful thinking. It seems that a range of behaviors occur during problem solving that could, in general, be considered forms of insightful thinking. In prior research (e.g., Metcalfe \& Wiebe, 1987; Schooler, Ohlsson, \& Brooks, 1993), insightful problem solving has been examined at a general level, focusing on overall solution rates and solving times, without providing a finegrained analysis of the behaviors occurring during problem solving. A deeper analysis of participants' problemsolving behaviors may reveal that the cognitive processes utilized by participants are diverse and do not lend themselves to a simple insight/analysis dichotomy.

Over the years, there have been few direct analyses of the processes underlying insight (see Weisberg \& Suls, 1973, for an example). Metcalfe (1986a, 1986b) conducted several groundbreaking studies in which the processes underlying insight were examined by analyzing differences in participants' metacognitions during problemsolving and related tasks. In one study, Metcalfe (1986a) demonstrated that although participants could successfully predict their ability to answer trivia questions, they were less successful in predicting their ability to solve insight problems. In related research, Metcalfe and Wiebe (1987) demonstrated that participants were accurate in judging how close they were to the solution of analytic problems throughout the solving process. In contrast, participants were not aware of their proximity to the correct solution of insight problems until immediately prior to solving the problems. These results supported prior research (Metcalfe, 1986b) on insight problem solving, in which participants pursuing an incorrect solution judged their own progress favorably, whereas participants pursuing a correct solution judged their own progress unfavorably until the moments immediately preceding the solution. The results indicated that solutions to insight problems occurred unpredictably and, thus, supported the notion that insight was the outcome of a sudden process, as has been proposed by Scheerer (1963).

Although the research conducted by Metcalfe (1986a, 1986b; Metcalfe \& Wiebe, 1987) demonstrated that differences exist in participants' metacognitive experiences while solving insight problems, in comparison with other problem types, those studies did not provide specific information concerning the thought processes underlying insight. That is, Metcalfe's studies measured only overall solution rates and, therefore, did not demonstrate that 
the insight problems were actually solved through the insight sequence discussed by Ohlsson (1992). Thus, although Metcalfe's results are consistent with her participants' having gone through that sequence, we have no direct evidence that they actually did so. As was noted earlier, Perkins (1981) reported that the participants in his studies of problem solving sometimes solved insight problems using analytic strategies but still demonstrated Aha! experiences. Thus, Metcalfe's participants might have solved her insight problems using analytic strategies, but they still would have produced the patterns of warmth ratings that Metcalfe interpreted as support for the Gestalt view of sudden insight. Therefore, we need more than warmth ratings to be sure that we are studying insight.

Similarly, Lavric et al. (2000) examined EEG patterns produced as participants solved insight versus analytic problems. Differences were found in the activation patterns, leading the authors to conclude that those patterns represented differences in the mental processes underlying insight versus analytic modes of solution. However, as in the research of Metcalfe (1986a, 1986b), Lavric et al. made no attempt to demonstrate that their participants actually solved the insight problem differently than the analytic problem; they simply assumed that there must have been basic differences in the solution methods.

Knoblich et al. (1999; see also Knoblich, Ohlsson, \& Raney, 2001) used matchstick arithmetic problems to study predictions from Ohlsson's (1992) insight theory. The theory predicts that when a participant reaches an impasse, certain types of problems will be easier to solve than others, because of the nature of the restructuring required to break out of the impasse. As an example, consider the constraint relaxation form of restructuring (see Table 1; see also Ohlsson, 1992). The researchers proposed that problems that required changes in the numerical values within the matchstick equations (i.e., constraints with narrow applications) would be solved more quickly than problems that required changes in addition or subtraction signs (i.e., constraints with broader applications to a range of equations). The results supported the prediction of longer solving times for the relaxation or restructuring of constraints with broader applications, and the researchers concluded that they had provided support for Ohlsson's (1992) theory concerning how impasses are broken during the insight sequence. However, as with other studies in the field (e.g., Lavric et al., 2000; Metcalfe \& Wiebe, 1987), no measure was taken of the participants' fine-grained behaviors or thoughts as they worked on the problems. Without such information, the results of this research simply provide us with the information that some matchstick arithmetic problems are harder than others, and little about the cognitive processes involved. Kaplan and Simon (1990) did attempt a fine-grained analysis of insight by examining verbal protocols; however, none of their participants were able to solve the problem used in their study without hints, so the results tell us little about the cognitive processes involved in ordinary problem solving.

In addition to problems arising from a too-global approach in the examination of the processes underlying insight problem solving, there is also a fundamental issue that is often unaddressed in studies of insight. The use of insight problems in the study of insight can be an a priori distinction that actually may interfere with understanding problem solving via insight. Labeling a problem an insight problem or an analytic problem, as in prior research (e.g., Metcalfe \& Wiebe, 1987; Schooler et al., 1993), tells us nothing about the cognitive processes involved in solving those problems. Therefore, it is possible that solutions to problems labeled as insight problems might not come about via the insight sequence (i.e., impasse followed by restructuring) or that problems labeled as insight problems may not represent a homogeneous group of problems. Our intent in using the candle problem in the present research was to create a problemsolving situation in which problem solving via insight was possible.

In conclusion, at present there are no data available concerning the actual frequency of insightful solutions to the problems labeled as insight problems. It seems that in order to test theories of how insight occurs, we need direct evidence concerning the processes underlying the solution of insight versus analytic problems. If we continue to rely on relatively gross measures, such as solution rates or solving times, we will be unable to say whether or not we are actually studying insight (Weisberg, 1995).

One method that has been useful in elucidating the thought processes underlying other types of problem solving is the collection of verbal protocols (Ericsson \& Simon, 1993; Perkins, 1981). Ericsson and Simon suggested that verbal protocols can be used as data within the problem-solving domain. They indicated that instructing participants to think aloud does not disrupt the sequence of the participants' thought processes, provided that only items naturally attended to by the participants are verbalized. It is only when experimenters instruct participants to explain their thoughts, or to explain a specific component of the problem-solving process, that changes in the sequences of cognitive processes may occur. This is especially the case if it has not been made explicitly clear through instruction and practice that the participant should concentrate on talking to himself or herself and should not attempt to explain things to the experimenter (Ericsson \& Simon, 1993, p. 79).

Although concurrent verbalization, performed as talking to oneself, does not change the nature or sequence of thought processes, there are circumstances under which concurrent verbalization can slow the rate of cognitive processes (Ericsson \& Simon, 1993). Excessive prompting by experimenters, intended to facilitate participants' verbalization rates, can slow the rate of cognitive processing. Thus, it is important to ensure that excessive prompting is avoided during the collection of verbal pro- 
tocols. It has also been suggested by Perkins (1981) that training in the use of the think-aloud procedure is important in ensuring that valid representations of participants' thought processes are obtained.

Several researchers have examined the potential effects of verbalization on a number of problem-solving tasksfor example, the tower of Hanoi (Karat, 1983) and algebra problems (Flaherty, 1974). Although prior research on insight has included verbalization in the methodology (e.g., Bulbrook, 1932; Duncker, 1945; Weisberg \& Suls, 1973), the effect of verbalization on participants' thought processes has gone largely unexamined. The collection of verbal protocols has been an effective method used by experimenters to elucidate the thought processes that underlie a number of behaviors. Because verbalization has the potential of providing researchers with detailed information concerning participants' thought processes, it is critical to consider the use of verbal protocols collected during insight problem solving as data and to examine what can be gained from an analysis of these protocols (Ericsson \& Simon, 1993).

In research with potentially broad implications, Schooler et al. (1993) have reported that verbalization has an interfering effect on insight problem solving, which they called verbal overshadowing of insight. Schooler et al. have suggested that differences exist between insight and noninsight problems in susceptibility to such overshadowing, and they proposed that there are processes involved in solving insight problems that are not readily verbalizable. Thus, when participants verbalize during insight problem solving, they are likely to focus on processes that are easier to verbalize but are not necessarily helpful in solving the problem. Schooler et al. reported a reduction in solution rates for insight problems as a result of instructions to think aloud. Verbal overshadowing has also been found when participants were asked to verbalize in other domains, including, but not limited to, visual imagery (Brandimonte, Schooler, \& Gabbino, 1997) and memory for taste (Melcher \& Schooler, 1996), faces (Fallshore \& Schooler, 1995), and colors (Schooler \& Engstler-Schooler, 1990). Most domains affected by verbal overshadowing appear to be nonverbal in nature. Consistent with this idea is research that has demonstrated that performance in several verbal tasks is actually facilitated by concurrent verbalization. Examples of such tasks include analytical problem solving (Schooler et al., 1993) and memory for verbal statements (Schooler \& Engstler-Schooler, 1990).

However, it should be noted once again that, as with the other studies already cited, Schooler et al. (1993) made no attempt to gather evidence concerning the processes occurring during the solution of the various problems they examined. Therefore, their results do not necessarily demonstrate that verbalization interferes with insight, because we have no way of knowing that their participants actually solved the insight problems through the insight sequence. On the basis of Perkins's (1981) results, cited earlier, it is possible on logical grounds that the insight problems were solved using analytic strategies and that those particular analytic strategies, and not the insight sequence, were interfered with by verbalization.

The finding of verbal overshadowing of insight in problem solving is potentially of great importance, in at least two ways. First, the possibility of verbal overshadowing supports the existence of differences in the solution processes underlying insight versus analysis as modes of problem solving. Second, the occurrence of verbal overshadowing of insight removes verbal protocols as a valid methodology for gathering information about the processes underlying insight. Thus, it is important to evaluate the specific conclusions drawn from the studies of Schooler et al. (1993). Schooler et al. examined three insight problems: the triangle of coins (Metcalfe, 1986b), the dealer (Perkins, 1981), and the prisoner (Metcalfe \& Wiebe, 1987). The researchers noted in Experiment 3 that the three insight problems they chose were selected specifically because they were most sensitive to effects stemming from concurrent verbalization, as was indicated in Experiments 1 and 2 of the same research. Therefore, the question arises as to the generality of verbal overshadowing of insight in problem solving. In addition, Schooler et al. presented their participants with limited instructions concerning the verbalization procedure, including a single training problem in which the participants were made familiar with the verbal protocol procedure. This preparation might not have been sufficient to discourage the participants' inclination to explain their cognitive processes and to encourage them simply to talk aloud as if to themselves (Ericsson, 2002; Perkins, 1981).

Ericsson (2002; see also Meissner, Brigham, \& Kelley, 2001) has indicated that the nature of the verbalization that participants are to engage in is a critical determinant of any effects that may stem from concurrent verbalization. Ericsson proposed that the information provided during concurrent verbalization is likely to be closest to underlying thoughts when the participant is instructed to verbalize thoughts that are already the focus of attention. Furthermore, it is important that the participants avoid explaining their thoughts, because explanation has been linked to increased effects from verbalization.

Research conducted by Meissner et al. (2001; see also Meissner \& Brigham, 2001) has also supported the important role that instructions play in the occurrence of verbal overshadowing. Meissner et al. examined participants' accuracy in the correct identification of a previously presented face in a subsequent lineup. The participants were shown the photograph of the target individual and then were asked to provide a verbal description of that target. Task instructions differed in terms of reporting instructions, with some instructions focusing on the importance of reporting only accurate information in their verbal descriptions and others focusing on the importance of providing thorough descriptions. Identification accuracy during the lineup was higher for the participants instructed to provide verbal descriptions of the target indi- 
vidual that focused on relaying only accurate information. In fact, when accuracy was the focus, subsequent lineup identification was as accurate as in the control condition, in which no verbal descriptions were provided. A meta-analysis conducted by Meissner and Brigham further supports the influence of instructions on the strength of the verbal overshadowing effect, with instructions that focused on elaboration during verbalization leading to stronger verbal overshadowing effects than did instructions that focused on free recall. The research of Meissner et al. and of Meissner and Brigham demonstrates the important influence of verbalization instructions on the occurrence of verbal overshadowing.

\section{Present Research}

If verbal overshadowing is found broadly across insight problems, the use of verbal protocols as data in the study of insight problem solving may be questioned. However, if the effect does not extend to other insight problems, it is possible that some factor other than the nature of the problems as insight problems may be implicated in Schooler et al.'s (1993) verbal overshadowing results.

The first goal of the present research was to determine whether the verbal overshadowing effect observed by Schooler et al. (1993) extends to Duncker's (1945) candle problem (see the Appendix). For almost 60 years, the candle problem has been part of the study of insight (e.g., Adamson, 1952; Duncker, 1945; Glucksberg \& Weisberg, 1966; Scheerer, 1963; Weisberg \& Suls, 1973); however, these studies have not directly examined the processes underlying insight. The candle problem is often cited in discussions of insight in the problem-solving literature (e.g., Ohlsson, 1992; Sternberg \& Davidson, 1995), as well as in psychology texts (e.g., Bernstein, Clarke-Stewart, Penner, Roy, \& Wickens, 2000; Westen, 1999). Therefore, the use of the candle problem seemed appropriate to examine the generality of verbal overshadowing.

The second goal of the present research was to determine whether additional data could be gathered about how participants solve problems labeled as insight problems. Ohlsson (1992) has proposed that impasse followed by restructuring is fundamental to the insight sequence. Given this sequence, then, insightful solutions result only when a problem solver becomes "stuck" while working on the problem and is able to break out of impasse via restructuring, which results in the solution to the problem. However, Weisberg (1995) has presented a different view of insight. According to Weisberg, impasse is not a critical component in the solution of insight problems. Insight problems can be solved via a series of restructurings that stem from additional knowledge about the problem situation gained by the individual during the solving process. For example, a participant might begin solving the candle problem by attempting to use the tacks to directly attach the candle to the door. If the participant's idea fails, because the tacks are too short, the participant gains the additional knowledge that directly attaching the candle to the door with the tacks will likely result in failure (Weisberg \& Suls, 1973). This new knowledge could change the participant's initial representation of the tacks as the only object needed in the solution. The participant might then consider the need for a ledge to support the candle. He or she might attempt to use the matchbook to create a ledge, but in doing so, it might be too flimsy to support the candle. Through this failed attempt, the participant gains the knowledge that a sturdier ledge is needed. This new information could lead to a search for a sturdier object that could serve as a platform - that is, a change in the goal — which could lead to the box. This example demonstrates, then, that without the experience of impasse, participants' representations may undergo multiple restructurings during the solving process.

If verbalization does not affect solution processes in the candle problem, it will be possible to use verbal protocols to determine whether the classic characteristics of insight (i.e., impasse and restructuring) are seen in the behavior of participants solving the candle problem. Furthermore, it will be possible to use verbal protocols to indicate the frequency of Ohlsson's (1992) insight sequence, as well as the frequency of solutions that occur neither through strict insight nor through analysis.

In examining performance on the candle problem, we predict that solutions to the candle problem will arise via modes of thinking other than strict analysis or insight, as defined by Ohlsson's (1992) insight sequence. Thus, we propose that the present research will demonstrate that multiple solution types occur when participants attempt to solve this problem.

\section{METHOD}

\section{Participants}

Fifty-eight Temple University undergraduates received course credit in an introductory psychology course for their participation in this research. Data from 6 participants were eliminated: Two participants failed to follow the task instructions; 2 reported prior exposure to the problem, and two verbal protocols were lost because of technical difficulties in sound recording. This resulted in a final sample of 52, of which 19 were male and 33 were female (mean age $=20.5$ years)

\section{Materials}

The test problem and two training problems used in this research can be found in the Appendix. When solving the candle problem, the participants were presented with a candle $(11.4 \mathrm{~cm}$ long and $3 \mathrm{~cm}$ in diameter), a book of matches, a box of thumbtacks $(7.6 \mathrm{~cm}$ long $\times 6.4 \mathrm{~cm}$ wide $\times 2.5 \mathrm{~cm}$ deep), and a piece of corkboard (61 $\mathrm{cm}$ long $\times 47 \mathrm{~cm}$ wide) that served as a door during the course of the experiment.

\section{Procedure}

The participants were tested individually in 1-h videotaped sessions. All the participants were exposed to the candle problem as part of a randomly ordered set of insight problems; results for only the candle problem are presented here. Other problems in the set included the triangle of coins (Metcalfe, 1986a), the socks (Sternberg \& Davidson, 1982), the lilies (Sternberg \& Davidson, 1982), the trees (Metcalfe, 1986a), and the necklace (Metcalfe, 1986b). 
Because this is the first attempt to perform a fine-grained analysis of participants' solving processes on a relatively wide range of problems, each problem deserves to be analyzed in detail. Presenting detailed analyses of performance on the remaining problems would have added substantial length to the article.

The participants were randomly assigned to either the verbalization condition $(\mathrm{V} ; n=34)$ or the nonverbalization condition $(\mathrm{NV}$; $n=18$ ). Participants in the $\mathrm{V}$ condition were instructed to solve all problems while thinking aloud, which was intended to provide a record of their thought processes. The NV condition served as the control condition. The participants were assigned to conditions with the restriction that the sample size for the $\mathrm{V}$ condition was approximately twice as large as that for the NV condition, because of our interest in performing subsequent analyses on the participants' verbal protocols. Therefore, the $\mathrm{V}$ condition could potentially provide more data for analysis than would be available from the NV condition.

The participants in both conditions were informed that they would be asked to solve a series of problems. They were permitted to make as many attempts as needed to solve the candle problem and were informed that they would have $10 \mathrm{~min}$ to work on the problem. The experimenter encouraged the participants to continue working on the problem until they generated the box solution or until the $10 \mathrm{~min}$ had elapsed. The participants were provided with scratch paper and a pen to record their ideas during the session. Those participants in the $\mathrm{V}$ condition received training on a modified version of the think-aloud procedure of Perkins (1981, p. 33). Instructions in the $\mathrm{V}$ condition were as follows.

While solving the problems you will be encouraged to think aloud. When thinking aloud you should do the following: Say whatever's on your mind. Don't hold back hunches, guesses, wild ideas, images, plans or goals. Speak as continuously as possible. Try to say something at least once every five seconds. Speak audibly. Watch for your voice dropping as you become involved. Don't worry about complete sentences or eloquence. Don't over explain or justify. Analyze no more than you would normally. Don't elaborate on past events. Get into the pattern of saying what you're thinking about now, not of thinking for a while and then describing your thoughts. Though the experimenter is present you are not talking to the experimenter. Instead, you are to perform this task as if you are talking aloud to yourself. Because you are thinking aloud it is important that you signal the experimenter when reporting a potential solution to the problem by saying "Is this correct?" or "My solution to the problem is ..." Otherwise the experimenter will think that you are still talking to yourself.

The participants in both conditions were asked to solve two training problems intended to familiarize them with the task itself and to allow the participants in the $\mathrm{V}$ condition to become familiar with thinking aloud before the test problems were attempted.

Following the participants' completion of the training problems, the test problems were administered. While the participants worked on both the training and the test problems, the experimenter remained silent, with the following exceptions: (1) If the participants in the $\mathrm{V}$ condition were not speaking loudly enough to be heard clearly, they were prompted with "Could you please speak up," and (2) if they were not speaking regularly (i.e., verbalizing approximately every $15 \mathrm{sec}$ ), they were prompted to "Please continue speaking." An examination of the 34 verbal protocols revealed that the participants were prompted to speak louder an average of $0.53(S D=$ $0.79)$ times per protocol. Prompting that encouraged the participants to continue speaking occurred an average of $2.24(S D=2.12)$ times per protocol. The rate of prompting did not differ between box solvers $(M=0.50, S D=0.53)$ and nonsolvers $(M=0.54, S D=$ 0.86 ) for the prompt to speak louder $[t(32)=0.119, p=.906, d=$ .05 ; two-tailed] or between box solvers $(M=2.38, S D=1.85)$ and nonsolvers $(M=2.19, S D=2.23)$ for the prompt to continue speaking $[t(32)=0.210, p=.835, d=.09$; two-tailed].

The experimenter also informed the participants in both conditions whether their responses were correct or incorrect (the box so- lution was regarded as the correct solution, although it should be noted that the participants were able to successfully attach the candle to the door via other methods) and prompted the participants to continue generating solutions if they had not generated the box solution. When the participants provided an incorrect solution they were told, "That's incorrect." An examination of the protocols revealed that the participants were informed by the experimenter that a solution response was incorrect an average of $1.71(S D=1.57)$ times per protocol. The rate of prompting concerning incorrect solution responses did not differ significantly between box solvers $(M=1.00, S D=1.20)$ and nonsolvers $[M=1.92, S D=1.62$; $t(32)=1.48, p=.148, d=.62$; two-tailed]; however, the medium effect size suggests that some difference did exist between the conditions, although not to the level of statistical significance.

\section{RESULTS AND DISCUSSION}

Our analyses of the participants' problem-solving performance and verbal protocols were carried out in two stages. First, to determine whether verbalization adversely affected problem solving, we compared the solution rates, solving times, and types of solutions generated by the participants in the V and the NV conditions. Second, we examined data from the verbal protocols for the presence of impasse, restructuring, and failure. Rates of impasse during problem solving and the relationship between the occurrence of impasse and the production of the box solution were examined. We also examined rates of restructuring during problem solving and the relationship between restructuring and the production of the box solution. Finally, we examined the relation between failure and impasse and between failure and restructuring.

\section{Coding}

Verbalization versus nonverbalization. The protocols of the participants in the $\mathrm{V}$ condition were transcribed, and the solutions generated by the participants in both verbalization conditions were classified (all transcribed protocols used in the following analyses are available on the Web, at http://astro.temple.edu/ jfleck). To classify the solutions, the two authors independently viewed the collected videotapes and drew all successful solutions or solution attempts. The same solution was drawn for the participants who were able to successfully generate a solution idea and for the participants who attempted to construct that same solution idea but failed to do so successfully (e.g., a participant attempted to balance the candle on several tacks, but the candle fell from the tack construction).

In summarizing the data for analysis, solutions that did not meet the criteria proposed in the problem were eliminated. For example, several participants used items that were not presented as objects in the problem during the generation of solutions. Two participants attempted to use the sheet of paper on which the problem was written, and another attempted to stand the candle on the rim of the corkboard door. In addition, several participants abandoned the goal of the problem in order to generate what they considered to be intrinsically interesting constructions, rather than attempting to deal with the prob- 
TACK (Direct) - Tack(s) pushed through the candle to attach it directly to the door.

a)

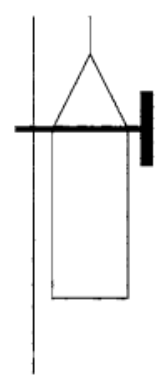

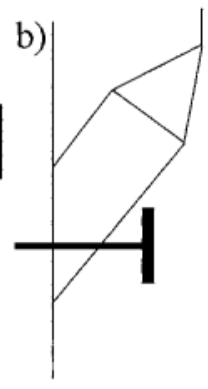

c)

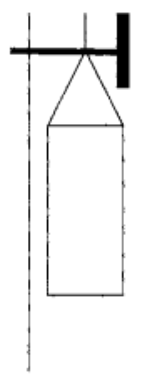

TACK (Hanger) - Multiple tacks used to create an interlocking configuration to hang the candle.

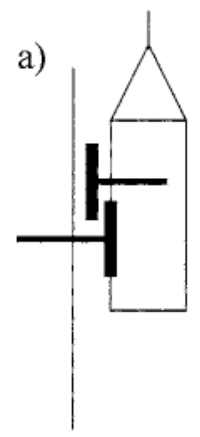

b)

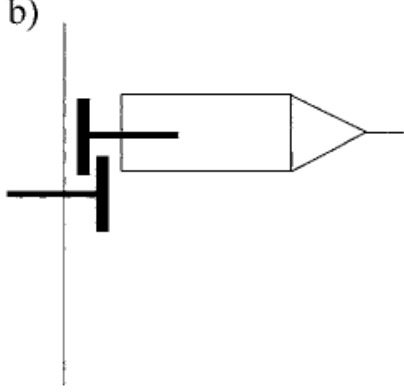

TACK (Holder) - Tack(s) used to create a holder for the candle.

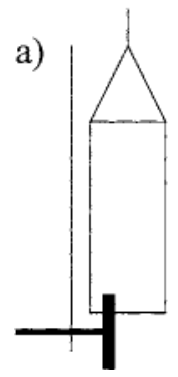

b)

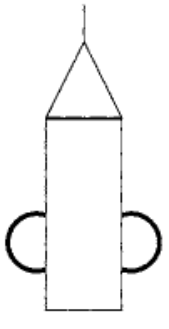

c)

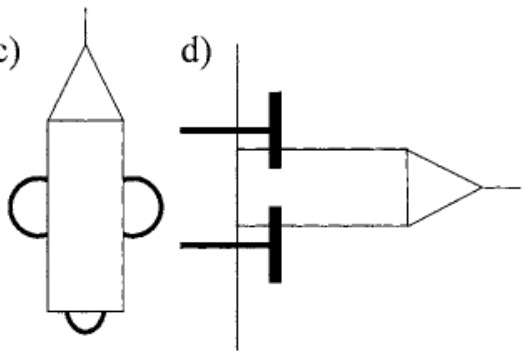

Figure 1. Examples of solution types.

lem itself (e.g., 1 participant created a caterpillar using the candle and some tacks). Approximately $12 \%$ of all solutions produced by the participants did not meet the problem parameters and were eliminated from further analysis. The two authors independently classified each solution according to the solution types listed in Figure 1 (examples of each solution type are also available). Interrater reliability (percentage of agreement), calculated for the classification of all solutions generated by the participants, was 93\%. Discussion was used to resolve any discrepancies in classification. The protocols were divided into segments by the authors by complete solution idea/attempt. That is, each new solution idea or attempt resulted in the beginning of a new segment.

Impasse, restructuring, and failure. In order to examine the presence of impasse, restructuring, and failure in the participants' verbal protocols, it was necessary to devise coding criteria for those phenomena that were appropriate to analysis of the candle problem. The quotation from Ohlsson (1992, p. 4) presented earlier provides descriptions of the types of behavior that signal the occurrence of an impasse. On the basis of Ohlsson's (1992) description, we identified a set of criteria that represented behaviors that typified impasse. Although it appears obvious from Ohlsson's (1992) definition that reaching a mental block and discontinuing behavior are essential for impasse, it should be noted that in collecting the participants' verbal protocols, they were asked to continue speaking throughout, making the complete cessation of behavior unlikely under the constraints of the task. Therefore, we generated a list of impasse criteria that allowed a more liberal assessment of impasse without the complete cessation of behavior. The list of criteria used during coding is presented in Table 2 . 
WAX - Wax is melted and used as an adhesive.

a)

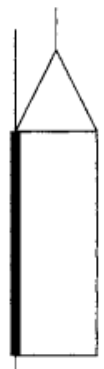

b)

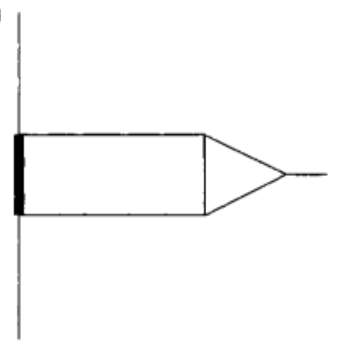

MATCHBOOK - Matchbook or matches from the matchbook are used to attach the candle to the door.
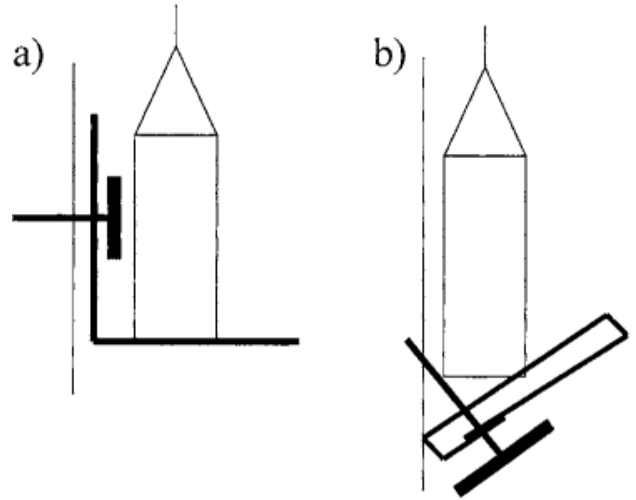

c)

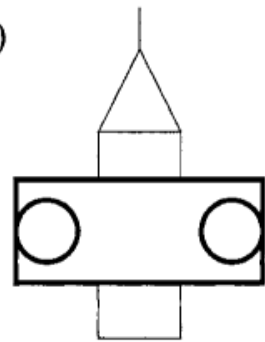

$\mathrm{BOX}-\mathrm{Box}$ is used to attach the candle to the door.

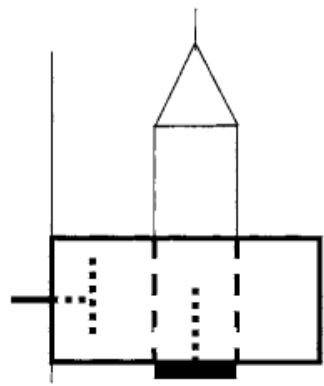

Figure 1 (Continued).

In order for a participant to meet the criteria for restructuring, his or her verbal protocol had to provide evidence of a change in problem representation. For the classification of restructuring data, we developed a set of coding criteria based on a classification scheme proposed by Ohlsson (1992). Ohlsson proposed three categories of restructuring that could be used by participants to break out of impasse: elaboration, reencoding, or constraint relaxation (see Table 1). We modified Ohlsson's (1992) classification scheme to fit the types of behaviors exhibited by participants working on the candle problem. We coded the protocols for the presence of restructuring on the basis of the following verbalizations and behaviors: (1) elaboration, where the participant finds a new function or use for an object; (2) reencoding, where the participant rejects the use of one object in the problem and uses another object that had not been considered previously; and (3) constraint relaxation, where the participant changes his or her representation of the goal state (i.e., the correct solution to the problem). Only examples of restructuring for which a change in thinking could clearly be determined from the protocols were included in the analysis. Therefore, a statement such as "Can I use these matches?" was considered an example of reencoding only if the participant either verbally discussed a plan to use the object in a solution or physically generated a solution involving that object.

In order for a participant's protocol to meet the criteria for failure, it had to be clear that the participant's solution idea or attempt was unsuccessful. We considered three potential sources of failure: experimenter rejection, failure of attempt, and inadequacy of idea/attempt. 
Table 2

Impasse Coding Criteria

\begin{tabular}{ll}
\hline Type 1: & Generating the same solution two or more times. \\
Type 2: & Rereading the problem three or more times in succession. \\
Type 3: & Indicating that his or her mind is blank. \\
& Indicating that he or she is out of ideas. \\
& Indicating that he or she is confused about the problem. \\
Type 4: & Guessing at the solution. \\
Type 5: & Demonstrating clear emotional frustration. \\
Type 6: & Demonstrating fixation on the inability to understand why a prior solution is incorrect. \\
Type 7: & $\begin{array}{l}\text { Discontinuing work on the problem-cessation of behavior. } \\
\text { Discontinuing verbalization. }\end{array}$ \\
& Not generating any additional solutions.
\end{tabular}

Note-A participant meets any criterion if it occurs one or more times in his or her protocol.

The category of experimenter rejection included all directives from the experimenter that indicated that a solution attempt was incorrect (the experimenter rejected all solutions other than the box solution). The category of failure of attempt included the participants' unsuccessful attempts to create some type of construction. Examples included the candle falling from the door, the wax of the candle breaking during an attempt to tack the candle to the door, and the tacks being too short to successfully attach the candle to the door directly, as in a Type 1 solution (see Figure 1). The third category, inadequacy of idea/attempt, was defined as a participant's rejecting a construction or an idea before actually carrying it out, because he or she found fault in the construction or idea and believed it would not result in the successful solution of the problem. For example, the participants rejected ideas because they realized that, if the idea were carried out, the candle would be too close to the door, the candle would be perpendicular to the door and, instead, should be parallel to it, or the candle needed to be attached directly to the door.

Two independent raters, blind to the hypotheses and the participants' solving success, coded all the protocols for the presence of impasse, restructuring, and failure. All information about solving success was removed from the protocols, so that the raters were not aware of which participants had produced the box solution. Furthermore, because other problems were administered, the coders were also blind to the participants' performance on the other problems in the problem set. Prior to independent coding, the coders worked with the first author, to ensure that they could reliably implement the coding scheme. Training continued until percentage of agreement between the coders reached $90 \%$.

When coding, the coders began at the beginning of a protocol and coded each segment for the presence of impasse, restructuring, and failure, using the classification schemes available in Tables 1 and 2, and as indicated in the aforementioned description of failure. As was noted above, protocols were segmented according to solution attempts or ideas. To label a segment as containing a type of impasse would require only the presence of that form of impasse in that segment, and not in subsequent sections of the protocol. The coders were specifically in- structed not to flip ahead when coding, which meant that they were not aware of the participants' solution success or lack thereof until reaching the end of the protocol.

Both raters found examples of only three types of impasse: (1) A participant indicated that he or she did not know what to do or was confused about the problem itself (Type 3), (2) a participant indicated that he or she did not understand why a prior solution was called incorrect by the experimenter (Type 6), and (3) a participant discontinued work on the problem (Type 7). Interrater reliability was calculated for the presence or absence of each of the three impasse types within all 34 protocols. The interrater reliability calculations (phi coefficients) for impasse were the following: Type $3, \phi=.82$; Type 6 , $\phi=1$; and Type $7, \phi=1$. Interrater reliability was calculated for each of the three types of restructuring in the 34 protocols, using phi coefficients. The interrater reliability calculations for restructuring were the following: reencoding, $\phi=.94$; elaboration, $\phi=.76$; and constraint relaxation, $\phi=.86$. Finally, interrater reliability was calculated for the three forms of failure, using phi coefficients. The interrater reliability calculations for failure were the following: experimenter rejection, $\phi=1$; failure of attempt, $\phi=.919$; and inadequacy of idea/attempt, $\phi=.847$.

\section{Analyses}

Verbalization versus nonverbalization. Eight participants in the $\mathrm{V}$ condition (23.5\%) and 5 participants in the NV condition $(27.7 \%)$ produced the box solution. The difference in box solution rates between the two conditions was not significant $\left[\chi^{2}(1, N=52)=0.113\right.$, $p=.736, \phi=.047]$. The $95 \%$ confidence interval of the effect size ranged from -.245 to .332 . An independentsamples $t$ test, comparing solving times between the $\mathrm{V}$ and the NV conditions, for box solvers only $(\mathrm{V}, M=5.97$ $\min , S D=3.70 ; \mathrm{NV}, M=5.32 \mathrm{~min}, S D=2.27$ ), was not significant $[t(11)=0.351, p=.732, d=.21$; twotailed]. The $95 \%$ confidence interval of the effect size ranged from -.37 to $.87 .{ }^{1}$

It is unwarranted to simply accept the null hypothesis when significant results are not obtained. Although differences were not found between the verbalization conditions in measures of solution rates or solving times, we 
thought it was necessary to determine whether there might be other differences between the $\mathrm{V}$ and the NV conditions. We next examined whether the verbalization conditions differed in the sequence of solution ideas generated by the participants. A chi-square analysis was calculated on the first solutions of the participants in the $\mathrm{V}$ and the NV conditions for the six solution types in Figure 1 . There were no differences between the two groups $\left[\chi^{2}(5, N=51)=0.890, p=.971\right] .{ }^{2}$ The contingency coefficient for this comparison was .131 (possible range, $0-.80$ ), indicating a similar distribution of data across the conditions (McNemar, 1962). The proportion of participants generating each solution type as their first solution is presented in Table 3. Approximately $75 \%$ of the participants used the tacks as the key component in creating the first solution, $14 \%$ used the wax from the candle, $6 \%$ used the matches/matchbook, and $4 \%$ used the box. The proportion of participants generating tackrelated solutions as their first solution is comparable to the results obtained in Weisberg and Suls (1973, Experiment 3 ), in which $83 \%$ of the participants utilized tacks in their first solution attempt.

We also examined possible differences in the first four solutions generated by the participants in the two conditions. We limited our analysis to the first four solutions because fewer than half of all the participants generated more than four solutions. The proportion of the participants' first four solutions for each solution type is presented in Table 3. ${ }^{3}$ The distributions of proportions for the two conditions are similar, with the largest difference between proportions being .08.

Because the differences between the two groups on all of the above measures were nonsignificant, the general solution data for both verbalizing conditions were collapsed across conditions. Overall, 25\% of the participants $(n=13)$ created the box solution; the average solving time was $5.72 \mathrm{~min}(S D=3.13)$. The box solution rates are comparable to those obtained in the prior research of Weisberg and Suls (1973) and Glucksberg and Weisberg (1966), who reported $25 \%$ solution rates. The similarity in solution rates in the present research to results obtained in prior research in which participants solved the problem without verbalizing further indicates that verbalization did not adversely affect the participants' solving processes.

Table 3

Proportion of Solution Types Generated by Participants in Each Verbalization Condition

\begin{tabular}{|c|c|c|c|c|}
\hline \multirow[b]{2}{*}{ Type } & \multicolumn{2}{|c|}{ First Solution } & \multicolumn{2}{|c|}{ First Four Solutions } \\
\hline & V & NV & V & NV \\
\hline Tack (direct) & .24 & .17 & .20 & .15 \\
\hline Tack (hanger) & .15 & .11 & .15 & .08 \\
\hline Tack (holder) & .39 & .44 & .33 & .38 \\
\hline Wax & .12 & .16 & .16 & .12 \\
\hline Matchbook/matches & .06 & .06 & .10 & .18 \\
\hline Box & .03 & .06 & .07 & .08 \\
\hline
\end{tabular}

In conclusion, no differences were found between the $\mathrm{V}$ and the NV conditions in the rates of producing the box solution, in solving times, or in the types of solutions generated during the overall solving process. This is contrary to the results reported by Schooler et al. (1993). According to their data, participants in the verbalization condition had lower solution rates than did participants in the nonverbalization condition for insight problems, but performed comparably to participants in the nonverbalization condition for noninsight problems. Schooler et al. concluded that the processes that underlie insight differ from those that are involved in solving noninsight problems and that, because those insight processes are not readily verbalizable, there is a verbal overshadowing effect on insight.

It is possible that methodological differences between the present study and that of Schooler et al. (1993) might have produced the differing results. First, the verbalization instructions used by Schooler et al. were brief and may not have been sufficiently clear in informing participants in the $\mathrm{V}$ condition that the nature of the verbalization was not to be explanatory. Prior research and reviews on the topic of concurrent verbalization (Ericsson, 2002; Meissner \& Brigham, 2001; Meissner et al., 2001) have suggested that the instructions used to elicit verbalization can significantly influence any effects that concurrent verbalization can have on task performance. Ericsson (2002; see also Ericsson \& Simon, 1993) has stressed the importance of verbalizing thoughts currently in attention, rather than explaining concepts. Furthermore, the research of Meissner and Brigham and of Meissner et al. has indicated that the verbal overshadowing effect indicated by Schooler et al. occurs only when participants are asked to generate extended verbalizations of visual stimuli. The failure by a number of researchers to replicate the verbal overshadowing effect (e.g., Meissner et al., 2001) indicates that the effect itself may be unstable.

In addition to the verbalization instructions, the single training problem used by Schooler et al. (1993) was a noninsight problem and may have influenced participants' expectations concerning the nature of the problem-solving task. That is, the participants may have concluded that all the test problems presented would be similar to the training problem, thereby limiting the range of their problem-solving strategies.

Because verbalization did not adversely affect performance on the candle problem according to any of our measures, we concluded that the participants' thought processes were not altered by verbalization during problem solving and that further analyses of the verbal protocols of the participants in the $\mathrm{V}$ condition were reasonable. These analyses could provide valuable information concerning the cognitive processes underlying insight. Therefore, we examined the verbal protocols for the presence of two key characteristics of the insight sequence-impasse and restructuring (e.g., Knoblich et al., 1999; Ohlsson, 1992) - and the role of failure in their occurrence. 
Impasse. We began our analysis of the insight sequence by examining the presence of impasse in the participants' verbal protocols. We found only 3 participants who demonstrated a full cessation of problem-solving behavior. Most of the participants continued to generate solutions throughout the solving period, without ever completely stopping all work. Therefore, we developed a classification scheme for coding impasse as either weak impasse or true impasse. A participant was identified as being in a state of weak impasse if he or she met one or more of the impasse criteria, excluding a cessation of behavior (Type 7; see Table 2). Only those participants who demonstrated the cessation of problem-solving behavior (i.e., the failure to generate solutions or to continue verbalizing after three or more successive prompts from the experimenter), with or without any other impasse criterion, were identified as exhibiting true impasse. As was noted earlier, we differentiated weak from true impasse on the basis of cessation of behavior, because of Ohlsson's (1992, p. 4) emphasis on that characteristic in his discussion of impasse.

Fifteen of the 34 protocols contained some form of impasse. Three of those 15 demonstrated a cessation of behavior, which clearly indicated that they were mentally at a loss (i.e., Type 7). When we examined the specific types of impasse demonstrated by the participants, little variability was exhibited (see Table 4). The majority of impasse-like behaviors fell under Type 3 impasse, with participants verbalizing that they lacked solutions, that their minds were blank, or that they were confused. Although there were participants who experienced Type 6 and Type 7 impasses, the occurrence of these behaviors was minimal.

In order to examine the role of impasse in the generation of the box solution, we examined the rates of production of the box solution in the participants who experienced impasse (weak or true) versus those who did not. Seven of the 19 participants who did not experience impasse (37\%) and 1 participant of the 15 who experienced impasse $(7 \%)$ produced the box solution. None of the 3 participants who experienced a complete cessation of solving behavior (i.e., true impasse) produced the box solution. A chi-square analysis was conducted on box solution rates for impasse (weak and true combined) versus nonimpasse participants. The difference between impasse and nonimpasse groups was significant $\left[\chi^{2}(1, N=\right.$ $34)=4.242, p=.039, \phi=.35]$. Thus, the participants who did not experience impasse during the solving period were more likely to go on to generate the box solution than were the participants who experienced impasse.

Several examples of weak impasse can be found in the following protocol:

I really have no idea so I'm just going to play because ... Actually have to attach it to the door ... There's no way you can do it that way-if someone can do it that way they'd have to be a genius because ... I don't ... So it can burn properly. Gosh, that doesn't make sense either . . . I'm thinking this is impossible because I can't ... What am I doing? I have no idea.

It is important to note that at no point when making these statements did the participant discontinue her work on the problem; she continued to generate nonbox solutions.

The examination of impasse allows us to draw several conclusions about its role in the candle problem. Most of the participants who experienced impasse verbalized that they were unsure of how to proceed with the problem or were out of ideas. They nonetheless continued to generate solutions to the problem, as evidenced by the 12 participants who only met the criteria for weak impasse. Furthermore, the participants who experienced impasse were unlikely to go on to produce the box solution. Although it seems logical that the participants who become stuck should not go on to solve the problem, it is important to consider what this finding suggests in terms of the insight sequence and, more generally, the study of insight. If it is the case that only solutions generated as a result of restructuring following impasse fall under the category of insightful solutions, we have little behavior of interest to study. In our analysis of the candle problem, $44 \%$ of the participants experienced some form of impasse, but only 1 individual, $3 \%$ of the 34 protocols analyzed, experienced impasse and then went on to generate the insightful solution.

It is possible, however, especially for the 3 participants who met the criterion for true impasse, that a $10-\mathrm{min}$ solving period did not provide enough time for the participants to break out of impasse and subsequently solve the problem in the bottom-up manner suggested by Ohlsson (1992). Although the 10-min solving period used in the present research is consistent with the 5-min to 10min solving periods used in prior research (e.g., Metcalfe \& Wiebe, 1987; Schooler et al., 1993; Weisberg \& Suls, 1973), it may be that longer solving periods are needed to observe the solution of the problem in response to true impasse. That possibility notwithstanding,

Table 4

Weak and True Impasse Frequencies

\begin{tabular}{lccc}
\hline \multicolumn{1}{c}{ Criteria } & $\begin{array}{c}\text { Weak* } \\
(n=12)\end{array}$ & $\begin{array}{c}\text { True* } \\
(n=3)\end{array}$ & $\begin{array}{c}\text { Combined } \\
(n=15)\end{array}$ \\
\hline Mind is blank (Type 3) & 12 & 2 & 14 \\
Not clear why prior work is incorrect (Type 6) & 2 & 1 & 3 \\
Failure to generate more ideas/solutions (Type 7) & 0 & 3 & 3 \\
\hline
\end{tabular}

*Several participants exhibited more than one impasse criterion. 
the present results do not provide strong support for Ohlsson's (1992) proposal that insights (defined in this situation as production of the box solution to the candle problem) occur in response to impasse.

Restructuring. The analysis of impasse uncovered few solutions to the candle problem following impasse. However, it is our view that solutions to the candle problem can arise independently of impasse, but still after restructuring (Weisberg, 1995; Weisberg \& Suls, 1973). These solutions would not be categorized by Ohlsson (1992) as exemplifying the insight sequence, but due to the presence of restructuring, such solutions should not be classified as stemming from analysis. We therefore examined the occurrence of restructuring in the participants' verbal protocols.

The presence of restructuring in the protocols was fairly common: Only 1 of the 34 participants failed to exhibit any restructuring during the solving period. All 8 of the participants who solved the problem exhibited one or more instances of restructuring in their protocols. The number of individual occurrences of restructuring by both box solvers and nonsolvers ranged from zero to eight $(M=3.15, S D=2.017)$. Restructuring can be found in the following protocol:

I have thumbtacks, which are not probably going to go through the candle. I might just be able to thumbtack them up on the door. Um, I could always try but that's not going to be right ... I only have thumbtacks. How am I supposed to, how is a candle supposed to stay up and burn with only thumbtacks? ... That won't work (trying to stick a tack in the top of the candle). If I stick the thumbtack through the candle the wax is just going to break off and it's not going to stay. And the matches, I might be able to do something with them ... [Not an example of restructuring - the potential use of the matches as a new object was never pursued.] If I make something for the candle that I can maybe place it on . . but I only have thumbtacks and I don't know how that's going to work. It could work. [Example of constraint relaxation - the participant was trying to attach the candle to the door directly but changed her conceptualization of the goal state in thinking that a ledge to support the candle might be a more effective solution.]

After several other restructurings, this participant generated the box solution.

We examined the relationship between the number of restructurings that occurred during a participant's problemsolving attempts and the subsequent production of the box solution. The point-biserial correlation calculated between the two variables was not significant $\left(r_{\mathrm{pb}}=\right.$ -.041 ), indicating no relationship between the number of restructurings during a participant's solution attempt and the subsequent production of the box solution.

To examine the relationship between impasse and restructuring, we conducted a one-way analysis of variance (ANOVA) to examine potential differences among the rates of restructuring under no impasse $(M=2.58, S D=$ $1.74)$, weak impasse $(M=4.50, S D=1.93)$, and true impasse $(M=1.33, S D=0.58)$ conditions. It should be noted, as was mentioned earlier, that the distinction between true and weak forms of impasse serves to represent the complete cessation of problem-solving behavior, as indicated in Ohlsson's (1992) definition (i.e., true impasse), as well as to provide a more liberal interpretation of impasse, given the verbalization requirements of the problem-solving task (i.e., weak impasse). The result of this analysis was significant $[F(2,31)=6.111, p=$ $.006]$. Tukey HSD post hoc analyses indicated significant differences in numbers of restructurings between true impasse and weak impasse conditions $(p=.024)$, as well as between no-impasse and weak conditions ( $p=$ .016), with weak impasse participants demonstrating more instances of restructuring than participants in the true or no-impasse conditions. Rates of restructuring, however, did not differ between participants in the noimpasse and the true impasse conditions $(p=.499)$.

We suggest that two components may contribute to this result. First, it is possible that the elevated levels of restructuring produced by the participants in the weak impasse condition may have stemmed from their rapid consideration of a number of alternatives in the hope of solving the problem. In other words, the participants in the weak impasse group generated more overall solution attempts. When a solution attempt was identified as inadequate or incorrect, the participants modified their thinking (i.e., produced a restructuring) and attempted additional solutions. Furthermore, their quicker progression through different solution attempts led to an exhaustion of their solution ideas, resulting in weaker forms of impasse. To test this possibility, we conducted a one-way ANOVA to examine potential differences among the number of unique solutions generated by the participants in the no-impasse $(M=2.53, S D=0.96)$, weak impasse $(M=3.50, S D=1.68)$, and true impasse $(M=1.00$, $S D=1.00)$ conditions. The result of this analysis was significant $[F(2,31)=5.261, p=.011]$. Tukey HSD post hoc analyses indicated significant differences in the number of unique solutions between true impasse and weak impasse conditions ( $p=.012)$, but not between weak impasse and no-impasse conditions $(p=.110)$. It should be noted, however, that the weak impasse condition did generate more unique solution attempts than did the other two conditions. Second, the lower rates of restructuring in the true impasse condition likely stemmed from the fact that the participants reached a cessation in problem-solving behavior, as indicated in the definition of true impasse, which might also have led to a decrease in the rates of restructuring.

Analyses of the restructuring data have led to several interesting conclusions about restructuring in the candle problem. First, it was possible to generate and reliably implement a classification scheme to test for the presence of restructuring. Second, restructuring was a common occurrence for the participants solving the candle problem, both for the participants who experienced impasse and for those who did not. Although the participants who experienced impasse also experienced re- 
structuring, the impasse and restructuring combination did not usually result in the insightful solution to the candle problem. Only 1 of the 14 participants who experienced both impasse and restructuring went on to solve the candle problem, using the box as a holder for the candle. It should be noted, however, that 7 of the 8 participants who generated the box solution experienced restructuring without impasse, a form of solving that does not meet the criteria for insight under the insight sequence of Ohlsson (1992). This mode of restructuring can be called top-down restructuring, because, in contrast to what we have called the bottom-up restructuring process postulated by Ohlsson (1992), in this case the process is being driven "from the top"- that is, by the person's knowledge and his or her analysis of the new information arising from the unsuccessful solution attempt. It is important here to differentiate bottom-up from top-down restructuring. In the latter, the individual changes the way of looking at the problem as a result of trying to deal with specific difficulties that have arisen in the problem (e.g., failure). In the former, as was noted earlier, the individual examines the situation without a plan, in the hope that something might open up possibilities for a new solution type.

Failure. We have suggested that failure during problem solving is related to the occurrence of restructuring (Weisberg, 1995; Weisberg \& Suls, 1973) and may be related to impasse as well. An examination of the protocols revealed that failure was a common occurrence (see Table 5), with a total of 213 instances of failure found. Of these, 67 (31\%) were instances of experimenter rejection, 97 (46\%) were instances of failure of attempt, 44 (21\%) were instances of inadequacy of idea or attempt, and $5(2 \%)$ were instances of failures that did not meet the criteria for the other categories (e.g., the solution is too dangerous to create).

In determining whether a relationship existed between failure and restructuring or between failure and impasse, we identified instances of failure that were followed by the occurrence of impasse and/or restructuring in a subsequent portion of the same segment of the protocol or in the segment immediately following. Of the 213 identified failures, $128(60 \%)$ were followed by impasse or restructuring, and $85(40 \%)$ were not. Of the $60 \%$ that were followed by impasse or restructuring, 10 instances of failure were followed by both impasse and restructuring. A binomial test indicated that significantly more failures were followed by impasse or restructuring than were not $(p=.004)$.

We then examined the presence or absence of failure prior to impasse and restructuring, independently. Instances of failure preceded impasse in 29 of the $30 \mathrm{im}-$ passe events that were identified (97\%; see Table 6A), demonstrating a close relationship between the two. In examining restructuring, instances of failure preceded restructuring in 99 of the 116 identified events $(85 \%$; see Table 6B). The specific frequencies for impasses and restructurings that occurred independently of failure are presented in bold (see Impasse Independent of Failure, Table 6A; Restructuring Independent of Failure, Table 6B).

We next considered more specifically the relationship between the type of failure and the type of restructuring or impasse that followed. For the instances of failure that were followed by impasse (see Table 6A), two trends emerged. First, experimenter rejection was more likely to result in impasse than were the other forms of failure. And second, for all forms of failure, the Type 3 form of impasse, where participants indicated not knowing what else to attempt, was most likely to follow. For instances of failure that were followed by restructuring, we determined the proportion of each restructuring type that followed each of the failure types. The resulting proportions indicated that the distributions of restructuring types were similar following the three failure types (see Table 6B).

The role of failure in the occurrence of impasse and restructuring is evident. The occurrence of failure was more likely to be followed by impasse or restructuring than not. Furthermore, both impasse and restructuring were more likely to occur following failure than independently. Impasse occurred independently of failure in only one case (3\%), suggesting that impasse is almost always preceded by an incident of failure; however, impasse occurred subsequent to failure in only 29 of the 213 instances of failure that were found $(14 \%)$, indicating that the occurrence of failure does not necessitate the occurrence of impasse. A greater percentage of failures were followed by restructuring (32\%) than by impasse; however, it is also true that there was a greater percentage of restructurings that occurred independently of failure $(15 \%)$ than of instances of impasse that occurred independently of failure (3\%). This result coincides with the results of Weisberg and Suls (1973), who found that failures of proposed constructions were critical in pro-

Table 5

Frequencies of Failures (General)

\begin{tabular}{lccr}
\hline \multicolumn{1}{c}{ Failure Type } & $\begin{array}{c}\text { Failure Followed by } \\
\text { Impasse or Restructuring }\end{array}$ & $\begin{array}{c}\text { Failure Independent of } \\
\text { Impasse or Restructuring }\end{array}$ & Total \\
\hline Experimenter rejection & 52 & 15 & 67 \\
Failure of attempt & 43 & 54 & 97 \\
Inadequacy of idea/attempt & 31 & 13 & 44 \\
Other & 2 & 3 & 5 \\
Total & 125 & 85 & 213 \\
\hline
\end{tabular}


Table 6A

Responses to Failure: Impasse

\begin{tabular}{lcllr}
\hline & \multicolumn{2}{c}{ Impasse Type Following Failure } & \\
\cline { 2 - 4 } \multicolumn{1}{c}{ Failure Type } & Type 3 & Type 6 & Type 7 & Total \\
\hline Experimenter rejection & $10(.62)$ & $4(.25)$ & $2(.12)$ & $16(1.0)$ \\
Failure of attempt & $6(1.0)$ & $0(0)$ & $0(0)$ & $6(1.0)$ \\
Inadequacy of idea/attempt & $6(.86)$ & $0(0)$ & $1(.14)$ & $7(1.0)$ \\
Other & 0 & 0 & 0 & 0 \\
$\quad$ Total failure $\rightarrow$ impasse & 22 & 4 & 3 & 29 \\
Impasse independent of failure & $\mathbf{1}$ & $\mathbf{0}$ & $\mathbf{0}$ & $\mathbf{1}$ \\
Total & 23 & 4 & 3 & 30 \\
\hline
\end{tabular}

Note-Proportions are shown in parentheses.

Table 6B

Responses to Failure: Restructuring

\begin{tabular}{lcccc}
\hline & \multicolumn{2}{c}{ Restructuring Type Following Failure } & \\
\cline { 2 - 4 } \multicolumn{1}{c}{ Failure Type } & & & Constraint & \\
& Elaboration & Reencoding & Relaxation & Total \\
\hline Experimenter rejection & $7(.19)$ & $11(.30)$ & $18(.50)$ & $36(1.0)$ \\
Failure of attempt & $15(.40)$ & $7(.19)$ & $15(.40)$ & $37(1.0)$ \\
Inadequacy of idea/attempt & $9(.38)$ & $7(.29)$ & $8(.33)$ & $24(1.0)$ \\
Other & 0 & 2 & 0 & 2 \\
Total failure $\rightarrow$ restructuring & 31 & 27 & 41 & 99 \\
Restructuring independent of failure & $\mathbf{8}$ & $\mathbf{4}$ & $\mathbf{5}$ & $\mathbf{1 7}$ \\
Total & 39 & 31 & 46 & 116 \\
\hline
\end{tabular}

Note-Proportions are shown in parentheses.

ducing the restructuring of the candle problem that we have called top-down restructuring. Recently, Ormerod, MacGregor, and Chronicle (2002) also noted that participants' failures to generate successful moves or constructions were essential to subsequent solution of the eight-coins problem, another problem presented in the literature as an insight problem.

In conclusion, we were able to identify a relationship between failure and restructuring, as well as between failure and impasse. Because the majority of restructurings were preceded by rejections of ideas or constructions, it seems reasonable to conclude that failure may play a critical role in structuring an environment so that the solution of insight problems is possible. We propose that failure in the candle problem could be an important contributor to the occurrence of top-down restructuring, because failure provides participants with new information about which solution ideas are likely to be effective and which are not. The importance of failure in the occurrence of restructuring has also been suggested in prior research with this problem (Weisberg \& Suls, 1973) and with others (e.g., Ormerod et al., 2002).

\section{CONCLUDING STATEMENTS}

The present study showed that instructing participants to verbalize did not adversely affect their thought processes when solving the candle problem. We thus failed to reproduce Schooler et al.'s (1993) verbal overshadow- ing effect. The present research, however, differs from the research of Schooler et al. in a number of ways. First, differences in verbalization's effect on problem solving may be linked to the types of insight problems used. If this is true, the three problems used in Schooler et al.'s research differ in some manner from the candle problem. Second, differences may stem from the manner in which the participants in the two studies executed the instructions to think aloud. Our instructions were specific about performing the task as if talking to oneself, rather than as if talking to the experimenter. And finally, other factors in the methodology may have contributed to differences in results. For example, it is possible that the types of training problems used in the two studies may have resulted in differences in the participants' task expectations and, therefore, problem-solving performance.

On the basis of the present study, it becomes clear that additional research is needed before we can adequately determine whether verbalization is an effective methodology for use with all problems labeled as insight problems or whether the implementation of this methodology is more appropriate for a subset of problems not affected by concurrent verbalization. First, it is important that the same insight and analytic problems as those used in Schooler et al.'s (1993) research be examined, using modifications to the methodology to determine whether those modifications influence results. Second, it will be important to examine the effects of verbalization in an expanded set of insight problems, to determine whether a 
verbal overshadowing effect occurs only in insight problems of certain types (e.g., riddles vs. manipulation problems; see Weisberg, 1995, for a review).

In the present research, analyses comparing the two verbalization conditions did not show significant differences. It, therefore, seems unlikely that instructing participants to verbalize while solving the candle problem adversely affects solution rates or solving times or results in changes in the underlying cognitive processes. The subsequent analysis of the participants' verbal protocols was useful in the identification of impasse and restructuring in participants solving the candle problem. We were able to find instances of impasse and restructuring in the verbal protocols of solvers and nonsolvers. In addition, the occurrence of restructuring was common in all the protocols, with the number of restructurings being unrelated to the production of the box solution. Impasse occurred less often, with the participants who experienced impasse being less likely to go on to produce the box solution. Although we identified 10 instances in the protocols where the occurrence of failure was followed by both impasse and restructuring, there was only 1 participant who produced the box solution after the occurrence of impasse and restructuring. The single occurrence of impasse in conjunction with restructuring, leading to the box solution, suggests that the insight sequence of Ohlsson (1992) occurs minimally in participants solving the candle problem. In addition, the number of solvers who generated the solution to the problem following multiple restructurings but without the experience of impasse demonstrates that the majority of solutions to the candle problem, an insight problem, do not occur via insight (i.e., impasse plus restructuring). The results of the present research suggest that there is a need to conduct deeper analyses of the processes involved in problem solving and that a basic insight/analysis di- chotomy is not sufficient to encompass the cognitive processes involved in problem solving.

A second issue facing researchers studying insight in problem solving is the stimuli used as insight problems. It has been suggested that a number of differences exist between insight and noninsight problems, including differences in the potential for restructuring during problem solving (i.e., a change in the problem representation vs. none), differences in the scope of individual problem spaces (i.e., the number of different solution paths available), and differences in the number of strategies that can lead to the solution of the problem (see Weisberg, 1995, for a review). What is less clear is how such distinctions between problems translate into differences in the actual occurrence of insight. For example, the candle problem may produce few instances of impasse's leading to restructuring and, therefore, solutions arising from Ohlsson's insight sequence, because there are many constructions that can be generated from the objects available (i.e., a large problem space). However, the marrying man problem (see the Appendix) may result in higher rates of true impasse in problem solvers, because the possible solution paths stemming from the problem itself are fewer in number. Support for this prediction can be found in the research of Ormerod et al. (2002), in which participants were more likely to achieve the solution to a problem when few potential solution paths existed for solving the problem.

The results of the present research suggest that the analysis of verbal protocols is an effective methodology through which to examine the cognitive processes underlying insight. Although researchers have examined many factors that contribute to insight (e.g., functional fixedness and incubation), few have attempted a more direct examination of the characteristics of cognitive processes theoretically implicated in reviews of insight

Table 7

A Continuum of Problem-Solving Methods: Stages in Problem Solving

1. Problem presented $\Rightarrow$ match with knowledge $\Rightarrow$ transfer solution (if no solution available $\Rightarrow \# 2$ )

A. If solution transfers $\Rightarrow$ problem solved (problem is familiar $\Rightarrow$ no Aha!)

B. If solution fails, but new information arises $\Rightarrow \# 3$

C. If solution fails and no new information arises $\Rightarrow \# 4$

2. Problem presentation $\Rightarrow$ No transfer $\Rightarrow$ Analysis $\Rightarrow$ Solution

A. No solution $\Rightarrow \# 4$

Comment: Person works through problem logically, trying to develop solution; if successful, problem solved directly; no impasse, no restructuring; however, Aha! is possible.

Top-Down Restructuring

3. New information from failure $\Rightarrow$ New match with knowledge $\Rightarrow$ New method (Restructuring)

A. If new method leads to solution $\Rightarrow$ stop

B. If new method leads to failure, but more new information arises from the failure $\Rightarrow \# 1$

C. If new method fails and no new information arises $\Rightarrow \# 4$

Comment: Restructuring based on feedback from problem; no impasse, but Aha! possible.

Ohlsson's Bottom-Up Restructuring

4. Impasse: "Switch when stuck?"

A. If bottom-up restructuring leads to new information $\Rightarrow \# 1$; if no new information arises $\Rightarrow$ stop

Comment: Attempt to acquire new information from bottom up, through reencoding, elaboration, and constraint relaxation 
problem solving. Therefore, the analysis of verbal protocols may be effective in evaluating existing theories of insight problem solving (e.g., Ohlsson, 1992), as well as testing the existence of the cognitive components believed to be fundamental components of insightful problem solving.

As a final point, it is important to note that the two views we have examined here-Ohlsson's (1992) postulated bottom-up restructuring in response to impasse and what we have called top-down restructuring in response to failure and new information-are not necessarily oppositional. Table 7 outlines a view of problem solving that assumes that there is a continuum of problem-solving methods between analysis and impasse, with Ohlsson's (1992) insight sequence being only one of several possible ways of solving problems. That is, one does not have to assume that solution to a problem involves either analysis (i.e., smooth progress) or impasse. One can solve an "insight problem" without impasse, but one still might not progress smoothly to solution. Furthermore, in carrying out a solution method intermediate between analysis and impasse, one can restructure a problem in a topdown manner and produce the insightful solution (Weisberg, 1980; Weisberg \& Suls, 1973).

The continuum view assumes that all problem solving begins with an attempt to match the problem with what one knows. If the person is familiar with the problem or with one like it, there is a solution available in memory that can be transferred to the new problem (Stage 1 in Table 7). ${ }^{4}$ If the method transferred from the old problem works in the new situation, the problem is solved (Stage 1A). If the transferred solution fails, there may be new information that becomes available from that failure (Stage 1B). This new information initiates an attempt to determine if the now-new problem is familiar (Stage 3), which can result in a top-down restructuring of the problem (i.e., restructuring of a problem without an impasse) and which could produce the insightful solution to an insight problem.

If Stage 3 is fruitless, the individual has reached an impasse (Stage 4). The bottom-up processes postulated by Ohlsson (1992) - elaboration, reencoding, and constraint relaxation - might then come into play. The individual examines various aspects of the problem, in the hope of discovering some new way of approaching it. However, it should also be noted that the question of how people respond to impasses is an empirical one; as the present study has demonstrated, it is not logically necessary that one respond to impasse with an attempt at bottom-up restructuring.

In conclusion, we suggest that the cognitive processes involved in problem solving should be viewed as a continuum between analysis and insight. By using protocol analysis in the study of problem solving, it will be possible to generate a more complete picture of the cognitive processes that occur during problem solving.

\section{REFERENCES}

Adamson, R. E. (1952). Functional fixedness as related to problem solving: A repetition of three experiments. Journal of Experimental Psychology, 44, 288-291.

Bernstein, D. A., Clarke-Stewart, A., Penner, L. A., Roy, E. J., \& Wickens, C. D. (2000). Psychology (5th ed.). New York: Houghton Mifflin.

Brandimonte, M. A., Schooler, J. W., \& Gabbino, P. (1997). Attenuation verbal overshadowing through color retrieval cues. Journal of Experimental Psychology: Learning, Memory, \& Cognition, 23, 915-931.

BulbrooK, M. E. (1932). An experimental inquiry into the existence and nature of "insight." American Journal of Psychology, 44, 409453.

DunCKer, K. (1945). On problem-solving. Psychological Monographs, 58(5, Whole No. 270).

ERICSSON, K. A. (2002). Towards a procedure for eliciting verbal expression of non-verbal experience without reactivity: Interpreting the verbal overshadowing effect within the theoretical framework for protocol analysis. Applied Cognitive Psychology, 16, 981-987.

Ericsson, K. A., \& Simon, H. A. (1993). Protocol analysis: Verbal reports as data (rev. ed.). Cambridge, MA: MIT Press.

FALlshore, M., \& SchoOler, J. W. (1995). Verbal vulnerability of perceptual expertise. Journal of Experimental Psychology: Learning, Memory, \& Cognition, 21, 1608-1623.

FLAHERTY, E. G. (1974). The thinking aloud technique and problem solving ability. Journal of Educational Research, 68, 223-225.

GARDNER, M. (1978). Aha! Aha! insight. New York: Scientific American.

GluCKSBERG, S., \& WeISBERG, R. W. (1966). Verbal behavior and problem solving: Some effects of labeling in a functional fixedness problem. Journal of Experimental Psychology, 71, 659-664.

Kaplan, C. A., \& Simon, H. A. (1990). In search of insight. Cognitive Psychology, 22, 374-419.

KARAT, J. (1983). Modeling learning and transfer in a problem solving task. Unpublished doctoral dissertation, University of Colorado at Boulder.

Knoblich, G., Ohlsson, S., Haider, H., \& Rhenius, D. (1999). Constraint relaxation and chunk decomposition in insight problem solving. Journal of Experimental Psychology: Learning, Memory, \& Cognition, 25, 1534-1555.

KNOBLICH, G., OHLSSON, S., \& Raney, G. E. (2001). An eye movement study of insight problem solving. Memory \& Cognition, 29, 10001009.

KöHLER, W. (1959). The mentality of apes. New York: Vintage Books. (Original work published 1925)

KöHLER, W. (1969). The task of Gestalt psychology. Princeton, NJ: Princeton University Press.

LAVRIC, A., ForstmeIER, S., \& RiPPON, G. (2000). Differences in working memory involvement in analytical and creative tasks: An ERP study. NeuroReport, 11, 1613-1618.

LuCHINS, A. S. (1942). Mechanization in problem solving: The effect of Einstellung. Psychological Monographs, 54(6, Whole No. 248).

LUNG, C. T., \& DominowsKI, R. L. (1985). Effects of strategy instructions and practice on nine-dot problem solving. Journal of Experimental Psychology: Learning, Memory, \& Cognition, 11, 804-811.

McNemar, Q. (1962). Psychological statistics (3rd ed.). New York: Wiley.

Meissner, C. A., \& Brigham, J. C. (2001). A meta-analysis of the verbal overshadowing effect in face identification. Applied Cognitive Psychology, 15, 603-616.

Meissner, C. A., Brigham, J. C., \& Kelley, C. M. (2001). The influence of retrieval processes in verbal overshadowing. Memory \& Cognition, 29, 176-186.

MELCHER, J. M., \& Schooler, J. W. (1996). The misremembrance of wines past: Verbal and perceptual expertise differentially mediate verbal overshadowing of taste memory. Journal of Memory \& Language, 35, 231-245. 
Metcalfe, J. (1986a). Feeling of knowing in memory and problem solving. Journal of Experimental Psychology: Learning, Memory, \& Cognition, 12, 288-294.

METCALFE, J. (1986b). Premonitions of insight predict impending error. Journal of Experimental Psychology: Learning, Memory, \& Cognition, 12, 623-634.

Metcalfe, J., \& Wiebe, D. (1987). Intuition in insight and noninsight problem solving. Memory \& Cognition, 15, 238-246.

Newell, A., \& Simon, H. A. (1972). Human problem solving. Englewood Cliffs, NJ: Prentice-Hall.

OHLsson, S. (1984). Restructuring revisited: II. An information processing theory of restructuring and insight. Scandinavian Journal of Psychology, 25, 117-129.

OHLsson, S. (1992). Information-processing explanations of insight and related phenomena. In M. T. Keane \& K. J. Gilhooly (Eds.), Advances in the psychology of thinking (Vol. 1, pp. 1-44). New York: Harvester Wheatsheaf.

Ormerod, T. C., MacGregor, J. N., \& Chronicle, E. P. (2002). Dynamics and constraints in insight problem solving. Journal of Experimental Psychology: Learning, Memory, \& Cognition, 28, 791799.

Perkins, D. (1981). The mind's best work. Cambridge, MA: Harvard University Press.

Scheerer, M. (1963). Problem solving. Scientific American, 208, 118128.

Schooler, J. W., \& EngSTLER-Schooler, T. Y. (1990). Verbal overshadowing of visual memories: Some things are better left unsaid. Cognitive Psychology, 22, 36-71.

ScHOOLER, J. W., OHLSSON, S., \& BROOKS, K. (1993). Thoughts beyond words: When language overshadows insight. Journal of Experimental Psychology: General, 122, 166-183.

StERnBerG, R. J., \& DAVIDSON, J. E. (1982). The mind of the puzzler. Psychology Today, 16, 37-44.
Sternberg, R. J., \& Davidson, J. E. (1995). The nature of insight. Cambridge, MA: MIT Press.

WeIsBerg, R. W. (1980). Memory, thought, and behavior. New York: Oxford University Press.

WeISBERG, R. W. (1993). Creativity: Beyond the myth of genius. New York: Freeman.

Weisberg, R. W. (1995). Prolegomena to theories of insight in problem solving: A taxonomy of problems. In R. Sternberg \& J. Davidson (Eds.), The nature of insight (pp. 157-196). Cambridge, MA: MIT Press.

WeIsBerg, R. W., \& AlbA, J. W. (1981). An examination of the alleged role of "fixation" in the solution of several "insight" problems. Journal of Experimental Psychology: General, 110, 169-192.

WEISBERG, R. W., \& SuLS, J. (1973). An information-processing model of Duncker's candle problem. Cognitive Psychology, 4, 255-276.

Wertheimer, M. (1961). Productive thinking (rev. ed.). London: Tavistock. (Original work published 1945)

Westen, D. (1999). Psychology: Mind, brain, and culture (2nd ed.). New York: Wiley.

\section{NOTES}

1. It should be noted that Ericsson and Simon (1993) have indicated that although providing verbal protocols does not alter thought processes, concurrent verbalization can slow the rate of cognitive processes, which would translate into longer solving times.

2. It should be noted that 1 participant did not produce any solutions.

3. Further analyses were not possible, because the observations were not independent.

4. An individual may have more than one possible solution available to a problem. When this occurs, several additional cycles through the stages in Table 7 will occur. However, since the overall logic of the solution process does not change, we will, for the sake of simplicity, assume that only one solution possibility occurs to the person.

\section{APPENDIX \\ Test and Training Problems}

\section{Test Problem}

The Candle

Problem. Using the items on the table, attach a candle to a door so that it can burn properly. Among the available objects are a book of matches and a box of tacks.

Solution. Use the box from the tacks as a candleholder or shelf.

Source. Duncker (1945).

\section{Training Problems}

\section{The Marrying Man}

Problem. A man in a town married 20 women in the town. He and the women are still alive, and he has had no divorces. He is not a bigamist and is not a Mormon, and yet he broke no law. How is that possible?

Solution. The man is the minister who married the women to their husbands.

Source. Gardner (1978).

The Matchsticks

Problem. Using six matches, make four equilateral triangles, with one complete match making up the side of each triangle.

Solution. Form a tetrahedron (a triangular-based pyramid) with the matchsticks.

Source. Scheerer (1963). 